\title{
Method offers DNA blueprint of a single human cell
}

\section{Easier cell-to-cell comparisons hold potential insights into cancer and other biological processes.}

\section{Monya Baker}

20 December 2012

Humans, strawberries, honeybees, chickens and rats are among the many organisms to have their DNA sequenced. But although sequencing an individual species is challenging, it is much harder to sequence the DNA of a single cell.

To get enough DNA for sequencing, thousands or even millions of cells are usually required. And finding out which mutations are in which cells is almost impossible, and mutations present in only a few cells (like early cancerous cells) are hidden altogether.

But a technique reported today in Science ${ }^{1}$ provides a way to copy DNA so that more than $90 \%$ of the genome of a single cell can be sequenced. The method also makes it easier to detect minor DNA sequence variations in single cells and, so, to find

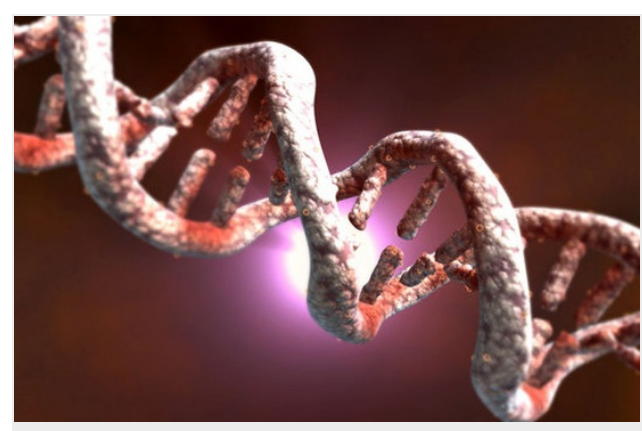

MEDICAL RF.COMSCIENCE PHOTO LIBRARY Huge numbers of cells are needed to sequence the DNA of a single cell. genetic differences between individual cells. Such differences can help to explain how cancer becomes more malignant, how reproductive cells emerge and even how individual neurons differ.

To sequence an individual cell, researchers must first make many copies of its DNA using techniques including PCR. But the drawback of these techniques is that some portions of the genome are copied much more extensively than others - a problem known as amplification bias - and this can drown out the least-copied areas of the genome making them undetectable. As a result, most attempts at single-cell sequencing cover, at best, around $70 \%$ of the genome evenly — although rates of around $40 \%$ are typical.

Sunney Xie, a chemical biologist at Harvard University in Cambridge, Massachusetts, and his colleagues have developed a technique, called multiple annealing and looping-based amplification cycles (MALBAC), that allows them to sequence $93 \%$ of the genome of a human cell. In MALBAC, DNA from a single cell is isolated, then short DNA molecules called primers are added. These are complementary to random parts of the DNA, which makes them stick to the strands and act as starting points for DNA replication.

The primers consist of two parts - a sticky eight-nucleotide portion that varies and binds to the DNA, plus a common sequence of 27 nucleotides. This common sequence stops the DNA from being copied too many times and massively cuts down the amplification bias. It does this by incorporating itself into the newly copied strands so that they loop back on themselves, which prevents over-copying.

\section{Easy recipe}

"MALBAC opens a door to many critical questions," says Bing Ren, who studies gene regulation at the University of California, San Diego. For example, it can be used to examine how quickly mutations accumulate, and to find variations in gene-copy number and chromosomal abnormalities across a population of cells. It also helps to detects variants across more of the genome than other sequencing methods.

"I think people are going to start using it right away," agrees James Eberwine, who works on single-cell genetics at the Perelman School of Medicine at the University of Pennsylvania in Philadelphia. He adds that researchers may have to tweak conditions - such as the ratio of primers to genomic DNA — to get experiments to work.

But although MALBAC covers the genome more thoroughly than other techniques, it is not perfect. It still misses perhaps one-third of single-nucleotide variations. Also, the enzyme that copies the DNA is error prone, so the copying process itself can introduce variants that were not present in the cell.

Xie was able to weed out all false positives, but only by comparing individually sequenced genomes from three closely related cells. That will increase costs, and could prove impossible for certain tissue samples, says Nicholas Navin at the MD Anderson Cancer Center in Houston, Texas, who has developed his own techniques for single-cell sequencing. 
Nature | doi:10.1038/nature.2012.12088

\section{References}

1. Zong, C., Lu, S., Chapman, A. R. \& Xie, X. S. Science 338, 1622-1626 (2012). 